\title{
Entanglement Detection by Current Measurements in Double Quantum Dot System
}

\author{
P. Busz*, P. RożeK, D. Tomaszewski and J. Martinek \\ Institute of Molecular Physics, Polish Academy of Science, 60-179 Poznań, Poland
}

\begin{abstract}
The development of quantum computing in quantum dots systems requires highly efficient and continuous solidstate source of spatially separated spin-entangled electrons. One of the approaches is a use of double quantum dot system connected to superconducting lead, where Cooper pairs provide a source of naturally entangled electrons. Apart from the source, an useful tool for detection of quantum entanglement is needed. We present entanglement detection by the ferromagnetic electrodes using entanglement witness operator method and direct measurement of spin polarized current in the system. We investigate requirements that have to be fulfilled by ferromagnetic detectors.
\end{abstract}

DOI: 10.12693/APhysPolA.127.490

PACS: 03.67.Mn, 03.67.Bg, 73.23.-b

\section{Introduction}

Quantum entangled states are an essential ingredient of quantum algorithms. Various physical systems can be used in order to obtain such states and manipulate them. However realizations in condensed matter physics seem to be the most promising since in these systems scalability can be achieved [1]. We investigate system consisting two quantum dots coupled to superconducting electrode and metallic electrodes or ferromagnetic detectors [2, 3], shown in the Fig. 1. In such system, superconducting electrode can serve as a source of naturally existing entangled states - Cooper pairs, which are in the singlet spin state. Electron pairs are able to tunnel and occupy the separate quantum dots while both of their spins preserve entanglement. Due to the use of entanglement witness method, an entanglement of electrons on the quantum dots can be confirmed, by only direct measurements of spin polarized current using ferromagnetic detectors.

\section{The Model Hamiltonian}

We consider double quantum dots (QDs) system where each dot has a single level $\varepsilon_{\eta}(\eta=L, R)$, and the Coulomb interaction $U$ between dots. We assume that a single QD cannot be doubly occupied, due to the strong on-site Coulomb interaction. We assume that a single QD cannot be doubly occupied, due to the strong on-site Coulomb interaction. The dots are coupled to a single superconducting and two ferromagnetic electrodes as depicted in Fig. 1. Therefore, the Hamiltonian of quantum dots can be given by:

$$
\hat{H}_{\mathrm{ddot}}=\sum_{\eta, \sigma} \varepsilon_{\eta, \sigma} \hat{n}_{\eta, \sigma}+U \sum_{\sigma, \sigma^{\prime}} \hat{n}_{L, \sigma} \hat{n}_{R, \sigma^{\prime}}
$$

where $\hat{n}_{\eta, \sigma}$ is the number operator of particles in a quan-

*corresponding author; e-mail: busz@ifmpan.poznan.pl

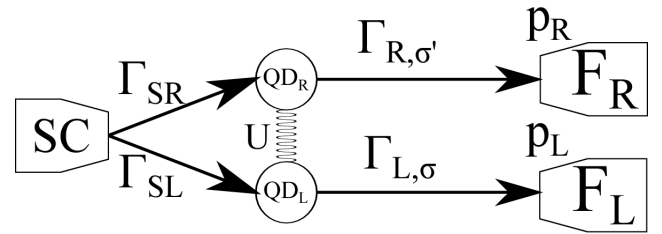

Fig. 1. Scheme of the studied system [4], where SC denotes superconducting electrode, $Q D_{\eta}$ quantum dots, and $F_{\eta}$ magnetic electrodes, where $\eta=R, L$. Voltage is applied to the ferromagnetic electrodes.

tum $\operatorname{dot} \eta$ and $U$ is the energy of the Coulomb interaction between the dots.

The tunneling between the dots and electrodes can be described by the following Hamiltonian:

$$
\begin{aligned}
& \hat{H}_{\mathrm{t}, F}=\sum_{\boldsymbol{k}, \sigma, \eta}\left(V_{F \eta, \sigma} c_{\eta, \boldsymbol{k}, \sigma}^{\dagger} d_{\eta, \sigma}+\text { H.c. }\right), \\
& \hat{H}_{\mathrm{t}, S}=\sum_{\boldsymbol{k}, \sigma, \eta}\left(V_{S \eta, \sigma} c_{S, \boldsymbol{k}, \sigma}^{\dagger} d_{\eta, \sigma}+\text { H.c. }\right),
\end{aligned}
$$

where $\mathrm{F} / \mathrm{S}$ denote the ferromagnetic and superconducting electrodes, respectively, $c_{\eta / S, k, \sigma}^{\dagger}$ are creation operators for electrons with momentum $k$ and spin $\sigma$ in $\mathrm{F} \eta / \mathrm{S}$ electrodes, $d_{\eta, \sigma}$ are annihilation operators for electrons with spin $\sigma$ in a QD $\eta$ and $V_{S / F \eta, \sigma}$ denotes the tunneling matrix amplitudes between the QDs and the superconducting/ferromagnetic electrodes. The effective spin polarization of ferromagnetic electrodes is defined as:

$$
p_{\eta}=\frac{\rho_{\eta, \uparrow}-\rho_{\eta, \downarrow}}{\rho_{\eta, \uparrow}+\rho_{\eta, \downarrow}},
$$

where $\rho_{\eta, \sigma}$ denotes the density of states at Fermi level for $\sigma=\uparrow, \downarrow$ majority / minority carriers and $\eta=L, R$ electrode. Then the coupling strengths are given by:

$$
\Gamma_{F \eta, \uparrow, \downarrow}=\frac{1}{2}\left(1 \pm p_{\eta}\right) \Gamma_{F \eta},
$$

where $\Gamma_{F \eta}=2 \pi\left(\rho_{\eta, \uparrow}+\rho_{\eta, \downarrow}\right)\left|V_{F \eta}\right|^{2}$ and $\Gamma_{S}=2 \pi \rho_{S}\left|V_{S}\right|^{2}$. 


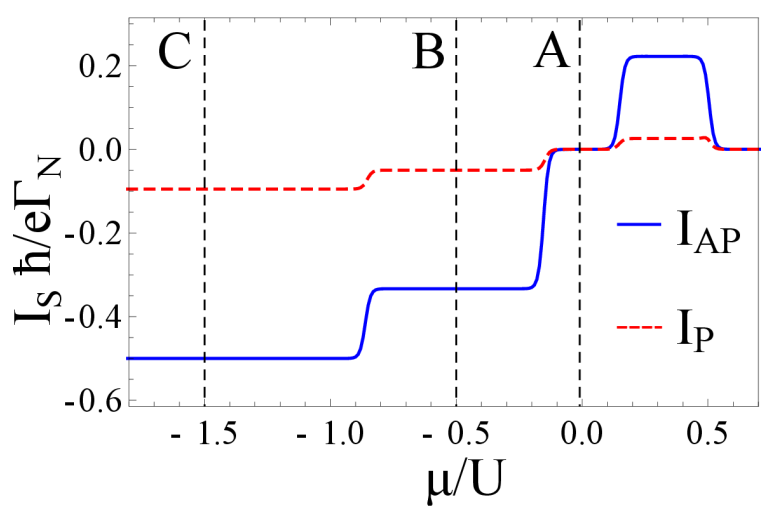

Fig. 2. The electric current $I_{S}$ as a function of the symmetric voltage $\mu_{L}=\mu_{R}=\mu$ for the symmetric system $\Gamma_{L}=\Gamma_{R}$ and degenerate dots levels $\epsilon_{L}=\epsilon_{R}$, spin polarization $p=0.9$ ( $\mathrm{P}$ - the parallel and AP - the antiparallel orientation of ferromagnetic lead magnetization) and detuning $\delta / U=0$. The temperature $k_{B} T=0.01 U$ and superconducting coupling $\Gamma_{S} / U=0.5$. Three distinctive point are marked: $\mathrm{C}$ at $\mu / U=-1.5, \mathrm{~B}$ at $\mu / U=-0.5$, A at $\mu / U=-0.01$ by dashed lines..

After tracing out the superconducting electrode degrees of freedom we obtain an effective Hamiltonian that takes into account the coupling to the superconducting lead [2]:

$$
\hat{H}_{\text {eff }}=\hat{H}_{\text {ddot }}-\frac{\Gamma_{S}}{2}\left(\hat{d}_{R, \uparrow}^{\dagger} \hat{d}_{L, \downarrow}^{\dagger}-\hat{d}_{R, \downarrow}^{\dagger} \hat{d}_{L, \uparrow}^{\dagger}+\text { H.c. }\right),
$$

where $\Gamma_{S}=\sqrt{\Gamma_{S L} \Gamma_{S R}}$ and the second term in Eq. (6) describes non-local proximity effect. The diagonalization of Hamiltonian, Eq. (6), leads to coupling between two states: $|0\rangle$ and $|S\rangle=\frac{1}{\sqrt{2}}\left(\left|\uparrow_{L} \downarrow_{R}\right\rangle-\left|\downarrow_{L} \uparrow_{R}\right\rangle\right)$ that results in new states:

$$
| \pm\rangle=\frac{1}{\sqrt{2}} \sqrt{1 \mp \frac{\delta}{2 \epsilon_{A}}}|0\rangle \mp \frac{1}{\sqrt{2}} \sqrt{1 \pm \frac{\delta}{2 \epsilon_{A}}}|S\rangle,
$$

where $\delta=\varepsilon_{R}+\varepsilon_{L}+U$ denotes a detuning parameter. Energies of states in the diagonal basis are: $E_{ \pm}=\delta / 2 \pm \epsilon_{A}$, $\epsilon_{A}=\sqrt{\delta^{2} / 4+\Gamma_{S L} \Gamma_{S R} / 2}$. That indicates that the nonlocal proximity effect changes the ground state of the system. Tunneling rates $W_{j i}$ between states $i, j$ can be determined from the Fermi golden rule $[2,5]$. Occupation probabilities obey the master equation:

$$
\sum_{i \neq j}\left(W_{i j} P_{j}-W_{j i} P_{i}\right)=0,
$$

where probabilities $P_{i}$ are normalized, $\Sigma_{i} P_{i}=1$. The current flowing in the electrode $\eta$ can be obtained from:

$$
I_{\eta}=\frac{e}{\hbar} \sum_{i \neq j} P_{i} W_{j i}^{\eta},
$$

where $e$ is the electron charge, the current rates $W_{j i}^{\eta}$ take into account the number of electrons transferred between lead $\eta$ and $Q D_{\eta}$.

The current calculated for different configurations of the ferromagnetic electrode magnetization is shown in Fig. 2. $I_{A P}$ denotes the current for the antiparallel alignment and $I_{P}$ for the parallel configuration. Suppression of the current in region $\mu / U>0.5$ results from the triplet blockade [2]. The current in the parallel configuration of electrodes is lower than in the antiparallel configuration. This corresponds to the fact that Cooper pairs are in spin-singlet states and the antiparallel alinement of electrode magnetizations better fits to the antiferromangetic order of the singlet state.

\section{Entanglement detection}

One way to detect quantum entanglement can be achieved by applying of the entanglement witness operator approach. Entanglement witness is an observable, for which an average value in a specific state is [6-8]:

$$
\begin{array}{ll}
\langle\hat{W}\rangle \geq 0 & \text { for all separable states, } \\
\langle\hat{W}\rangle<0 \quad \text { for some entangled states. }
\end{array}
$$

Entanglement witnesses for non-ideal $\left(p_{\eta}<1\right)$ ferromagnetic detectors can be postulated in a form:

$$
\hat{W}_{3 D}=\frac{1}{2}\left(\boldsymbol{I}+\left(\hat{M}_{x}+\hat{M}_{y}+\hat{M}_{z}\right)\right)
$$

and reduced to the two-dimensional $2 D$ case:

$$
\hat{V}_{i j}=\frac{1}{2}\left(\boldsymbol{I}+\left(\hat{M}_{i}+\hat{M}_{j}\right)\right),
$$

where $i, j \in\{x, y, z\}, \quad i \neq j$. Here the operators $\hat{M}_{i}$ $(i=x, y, z)$ can be related to the spin correlations by

$$
\hat{M}_{i} \propto \hat{\sigma}_{i} \otimes \hat{\sigma}_{i}
$$

and can be measured by the spin polarized electric current:

$$
\left\langle\hat{M}_{z}\right\rangle=\frac{I_{\uparrow \uparrow}+I_{\downarrow \downarrow}-I_{\uparrow \downarrow}-I_{\downarrow \uparrow}}{I_{\uparrow \uparrow}+I_{\downarrow \downarrow}+I_{\uparrow \downarrow}+I_{\downarrow \uparrow}} .
$$

Here $I_{i j}$ denotes the value of the current flowing through the system with the magnetization of $L$ electrode in $i$ direction, and of $R$ electrode in $j$ direction both among the $z$-axis. It can be proved that operators Eq. (11) and Eq. (12) are entanglement witnesses for the studied system since they fulfill the conditions given by Eq. (10). With use of obtained current-voltage characteristics, average values of entanglement witnesses (for several values of the voltage) are shown as a function of the spin polarization $p=p_{L}=p_{R}$ and symmetric coupling $\Gamma_{L}=\Gamma_{R}$ (Figs. 3 and 4 ).

The range of applicability of witness operators corresponds to the area where the average value of operator is negative. The minimal required spin polarization for entanglement detection can be read out from plots. Twodimensional entanglement witness requires a higher spin polarization of leads than three-dimensional.

\section{Summary}

We investigate the system of double quantum dot coupled with superconducting electrode acting as a source of entangled electrons and two ferromagnetic electrodes serving as spin-resolved detectors. The superconducting non-local proximity effect gives rise to coupling of singlet state with vacuum state, which resulted in non-local Andreev reflection. Therefore, spin entangled electron singlet states were induced on the dots. Using of the 


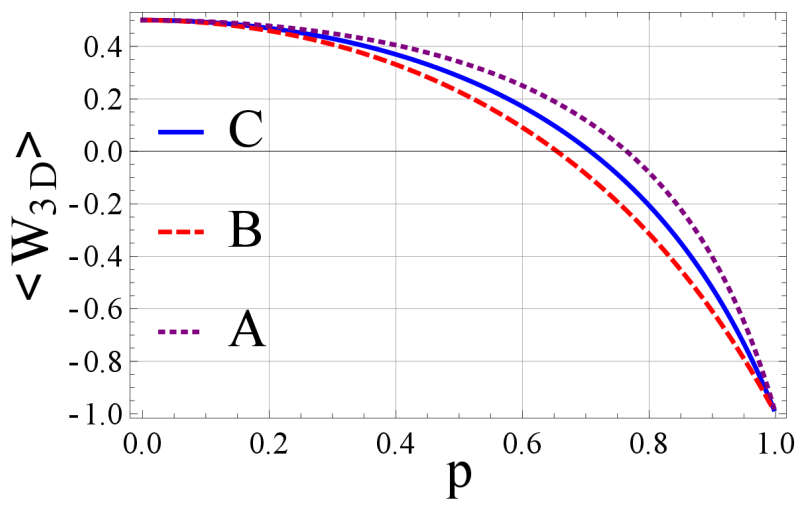

Fig. 3. 3D entanglement witness $\hat{W}_{3 D}$ for all three characteristic steps A, B, C marked on Fig. 2. Detection of entanglement is possible for spin polarization: $p(\boldsymbol{C}) \gtrsim 0.707, p(\boldsymbol{B}) \gtrsim 0.654, p(\boldsymbol{A}) \gtrsim 0.765$.

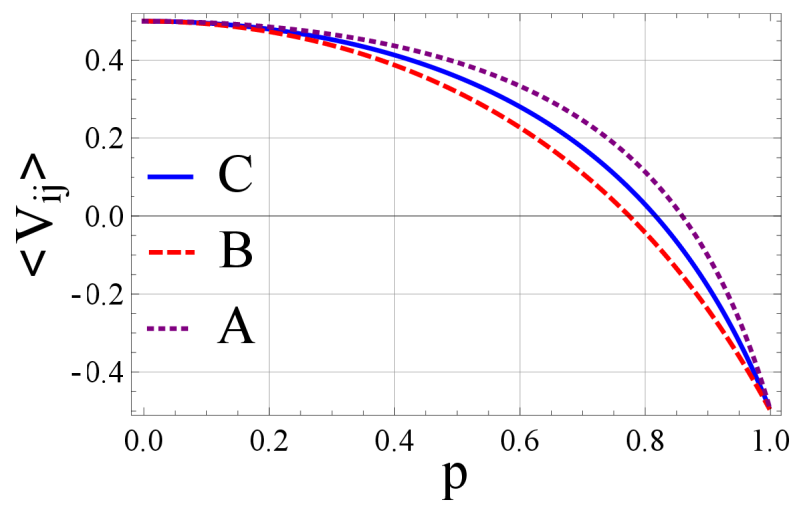

Fig. 4. 2D entanglement witness $\hat{W}_{2 D}$ for all three characteristic steps A, B, C marked on Fig. 2. Detection of entanglement is possible for spin polarization: $p(\boldsymbol{C}) \gtrsim 0.817, p(\boldsymbol{B}) \gtrsim 0.775, p(\boldsymbol{A}) \gtrsim 0.859$. Different witnesses $V_{i j}$ have identical form when averaging in singlet state.

entanglement witness method to our system we demonstrate how to detect the spin entanglement by the electric current measurements. The minimal required spin polarizations of ferromagnetic detectors for entanglement detection were determined. Point $\mathrm{B}, \mu / U=0.5$, corresponds to the lowest spin polarization $p=0.654$ required for the entanglement detection.

\section{Acknowledgments}

This work has been supported by the EUFP7 project SE2ND [271554] and Polish Grant for Science for the years 2010-2015.

\section{References}

[1] A. G. Fowler, M. Mariantoni, J. M. Martinis, A. N. Cleland, Phys. Rev. A. 86, 032324 (2012).

[2] J. Eldridge, M. G. Pala, M. Governale, J. König, Phys. Rev. B. 82, 184507 (2010).

[3] P. Trocha, J. Barnaś, Phys. Rev. B 89, 245418 (2014).

[4] L. Hofstetter, S. Csonka, J. Nygård, C. Schönenberger, Nature 461, 960 (2009).

[5] H. Grabert, M. H. Devoret, Single charge tunneling: Coulomb blockade phenomena in nanostructures, Plenum Press, 1992.

[6] M. Horodecki, P. Horodecki, R. Horodecki, Physics Letters A 223, 1 (1996).

[7] W. M. de Muynck, H. Martens, Phys. Rev. A. 42, 5079 (1990).

[8] W. Kłobus, A. Grudka, A. Baumgartner, D. Tomaszewski, C. Schönenberger, J. Martinek, Phys. Rev. B. 89, 125404 (2014). 